\title{
Persistence, Discontinuation, and Switching Patterns of Newly Initiated TNF Inhibitor Therapy in Ankylosing Spondylitis Patients in the United States
}

\author{
Theresa Hunter $\cdot$ Krista Schroeder $\cdot$ David Sandoval $\cdot$ Atul Deodhar
}

Received: January 2, 2019 / Published online: March 5, 2019

(C) The Author(s) 2019

\begin{abstract}
Introduction: The primary goals of treating ankylosing spondylitis (AS) patients are to maximize long-term health-related quality of life through control of symptoms and inflammation, prevention of progressive structural damage, and preservation of function. The objective of this study was to describe treatment patterns (persistence, discontinuations, and switch) in the 2 years following the initiation of tumor necrosis factor inhibitors (TNFi) therapy in AS patients.

Methods: Adult patients with $\geq 2$ AS diagnostic codes (ICD-9: 720.0 and/or ICD-10:M45.x) by a healthcare provider were included in this retrospective analysis of data from the IBM MarketScan Commercial Claims database. Patients who newly initiated a TNFi from 01/01/2009 to $12 / 31 / 2013$ were indexed on their first TNFi. Patients were required to have a 1-year pre-index period free of TNFi and continuous enrollment 1-year pre-index and 2-year post-index.
\end{abstract}

Enhanced Digital Features To view enhanced digital features for this article go to: https://doi.org/10.6084/ m9.figshare.7745729.

T. Hunter $(\bowtie) \cdot$ K. Schroeder $\cdot$ D. Sandoval Eli Lilly and Company, Indianapolis, IN, USA e-mail: hunter_theresa_marie@lilly.com

A. Deodhar

Oregon Health and Science University, Portland, OR, USA
Patients were excluded if they had $\geq 2$ diagnostic codes for any of the following conditions: rheumatoid arthritis, juvenile idiopathic arthritis, psoriatic arthritis, Crohn's disease, ulcerative colitis, plaque psoriasis, hidradenitis suppurativa, or uveitis. Demographic, clinical, and treatment patterns were analyzed. Treatment patterns included switching to a new TNFi, discontinuation ( $\geq 90$-day gap in therapy without starting a new TNFi), or persistence (no gaps in therapy $\geq 90$ days) during the 2-year follow-up period. Logistic regression analyses predicting persistent vs. non-persistent and switching vs. discontinuation were conducted.

Results: A total of 1372 AS patients (846 males/ 526 females) met the inclusion criteria for this study. Males had a mean age of 44.3 years, while females had a mean age of 42.3 years. Adalimumab was the first biologic for the majority of patients (44.6\% males $/ 43.3 \%$ females), followed by etanercept $(40.4 \%$ males $/ 41.6 \%$ females), infliximab (10.4\% males $/ 10.8 \%$ females), golimumab (4.6\% males $/ 3.8 \%$ females), and certolizumab pegol $(0.0 \%$ males $/ 0.4 \%$ females $)$. During the follow-up period, $33.1 \%$ of patients $(n=454)$ were persistent on their index TNFi, $40.7 \%(n=559)$ discontinued their index TNFi and did not restart a TNFi, and 26.1\% $(n=359)$ switched to a second TNFi. Patients prescribed cDMARDs were more likely to be persistent, while females and opioid users were less likely to be persistent on their first TNFi. Among those that discontinued their first TNFi, 32.8\% 
$(n=187)$ of males and $43.6 \%(n=177)$ of females switched to a second TNFi.

Conclusions: This study suggests that approximately $67 \%$ of male AS patients and $77 \%$ of female AS patients newly initiating a TNFi do not remain on the index therapy 2 year post initiation.

Funding: Eli Lilly and Company.

Keywords: Ankylosing spondylitis; Persistence; Treatment patterns

\section{INTRODUCTION}

Ankylosing spondylitis (AS) is a chronic immune-mediated inflammatory disease that predominantly affects the sacroiliac joints and spine [1]. AS is associated with pain, impaired health-related quality of life (HRQoL), and disability [2,3]. Musculoskeletal features as well as extra-articular manifestations such as enthesitis, uveitis, inflammatory bowel disease, and psoriasis can cause a substantial burden to AS patients.

Treatment options for AS patients include physical therapy, nonsteroidal anti-inflammatory drugs (NSAIDs), and biologics such as tumor necrosis factor inhibitors (TNFi) and interleukin-17 (IL-17) antagonists [4-10]. The American College of Rheumatology (ACR), Spondylitis Association of America (SAA), and Spondyloarthritis Research and Treatment Network (SPARTAN) recommend physical therapy plus NSAIDs as a first-line treatment for AS and biologic therapy for patients that do not respond to NSAIDs [10]. TNFi have demonstrated efficacy in the treatment of AS, however some patients do not respond to treatment (primary treatment failure) or experience diminished efficacy over time (secondary treatment failure), while others experience side effects that require treatment discontinuation [11].

The purpose of this study was to examine treatment patterns and therapy modifications in AS patients that are newly receiving TNFi therapy. This study also aimed to examine the characteristics of AS patients that are persistence and non-persistence (discontinuation and switching) of TNFi therapy.

\section{METHODS}

\section{Study Design}

This study was an observational, retrospective, cross-sectional study based on data from the IBM MarketScan ${ }^{\circledR}$ Research database. The setting for this study was US clinical practice, as reflected by the insurance claims in the database. IBM Marketscan Commercial, Medicaid and Medicare-Supplemental Claims database contains de-identified data on over 50 million covered lives and capture the continuum of care in all settings including physician office visits, hospital stays, and pharmacies. Study variables were defined in the IBM MarketScan ${ }^{\circledR}$ Research database using enrollment records and International Classification of Diseases, 9th Revision, Clinical Modification (ICD-9-CM) and International Classification of Disease, 10th Revisions, Clinical Modification (ICD-10-CM) codes. All data from the databases are Health Insurance Portability and Accountability Act (HIPAA) compliant to protect patient privacy.

AS patients aged 18 and over that had at least one claim for a TNFi (adalimumab, etanercept, infliximab, golimumab, or certolizumab pegol) during the index period (1/1/2009-12/31/2013) were included in this cohort study. The first TNFi claim during the study time period was considered the index period for this study. After the index date, each patient is then followed for 2 years. If patients did not have at least 2 years of follow-up, they were excluded from the study. The final follow-up for this study could be $12 / 31 / 2015$. Patients were considered to have AS if they had at least two diagnostic codes for AS (ICD-9-CM: 720.0 or ICD-10-CM: M45.x on any medical claim, either primary diagnosis or secondary diagnosis) by a physician during the study period. Patients were excluded if they had $2+$ diagnostic codes for any of the following conditions: rheumatoid arthritis, juvenile idiopathic arthritis, psoriatic arthritis, Crohn's disease, ulcerative colitis, plaque psoriasis, hidradenitis suppurativa, or uveitis. For this 
cohort study, patients had to have continuous enrollment (medical and prescription) 1 year pre-index and 2 years post-index. Up to 45 days of an enrollment gap was allowed (i.e., at least 1050 days of enrollment required). This study has been performed in accordance with the ethical standards as laid down in the 1964 Declaration of Helsinki and later amendments. This study was a retrospective analysis of administrative claims data and does not contain any human participants or animals. Thus, permission from a research ethics committee was not required and formal informed consent was not obtained. This study was designed and implemented in accordance with the Guidelines for Good Pharmacoepidemiology Practices of the International Society for Pharmacoepidemiology, the Strengthening the Reporting of Observational Studies in Epidemiology (STROBE) [22] guidelines, and the ethical principles laid down in the 1964 Declaration of Helsinki and its later amendments or comparable ethical standards. This study was a retrospective analysis of administrative claims data and does not contain any human participants or animals. Thus, this study was exempt from an institutional review board.

\section{Statistical Analyses}

Data from January 1, 2008 to December 31, 2015 was evaluated to determine treatment patterns (in a 2-year follow-up) of AS patients initiating on first-line TNFi from 01/01/2009 to $12 / 31 / 2013$. Treatment patterns included switching to new TNFi ( 2 and $3+$ lines), discontinuation, OR persistence during follow-up. Switching was defined as being prescribed a second (or third) TNFi during the 2-year followup. Given that TNFi are not recommended to be prescribed in combination, no other criteria (days overlap, no new script fill) were used to determine switching. Persistence/discontinuation was defined as a 90-day gap in therapy. Patients were stratified into the following three groups (based on the analysis above): (1) persistent on index TNFi, (2) discontinue index TNFi (switching not allowed), and (3) switch to a second TNFi during follow-up (but not a third). Patient demographic characteristics such as age, gender, geographic region, and insurance type were assessed at baseline (index) in the overall cohort and the subgroups.

Descriptive summary statistics of patient demographics and treatment patterns were conducted for the overall cohort and subgroups. Categorical variables were analyzed by frequency counts and percentages while continuous variables were analyzed by mean (SD).

Logistic regression analyses predicting persistence vs. non-persistence (switch or discontinuation) during the follow-up period. The following covariates at index were included in the logistic regression model: age (continuous); gender (male or female); and insurance type (commercial, Medicare-Supplemental, Medicaid). In addition, the following covariates (during the 1-year pre-index period) were also included in the logistic regression model: Charlson Comorbidity Index (CCI); emergency room (ER) visit; inpatient stay; rheumatologist visit; number of outpatient visits; conventional DMARD use (sulfasalazine and methotrexate); opioid use (codeine, oxycodone, hydrocodone, propoxyphene, dihydrocodeine, fentanyl, hydromorphone, levorphanol, methadone, morphine, oxymorphone, tramadol, tapentadol, meperidine, butorphanol, buprenorphine, nalbuphine, pentazocine); glucocorticoid use (betamethasone, cortisone, dexamethasone, hydrocortisone, methylprednisolone, prednisolone, prednisone, triamcinolone); and nonopioid analgesic use.

\section{RESULTS}

\section{Demographics and Baseline Characteristics}

A total of 1374 patients with AS met the inclusion criteria and were included in the analyses. The majority of patients were male $(61.7 \%)$ and the overall mean age was 43.8 years. Males had a mean age of 44.3 years, while females had a mean age of 42.3 years. The largest percentage of patients were receiving commercial insurance $(91.3 \%)$ (Table 1). 


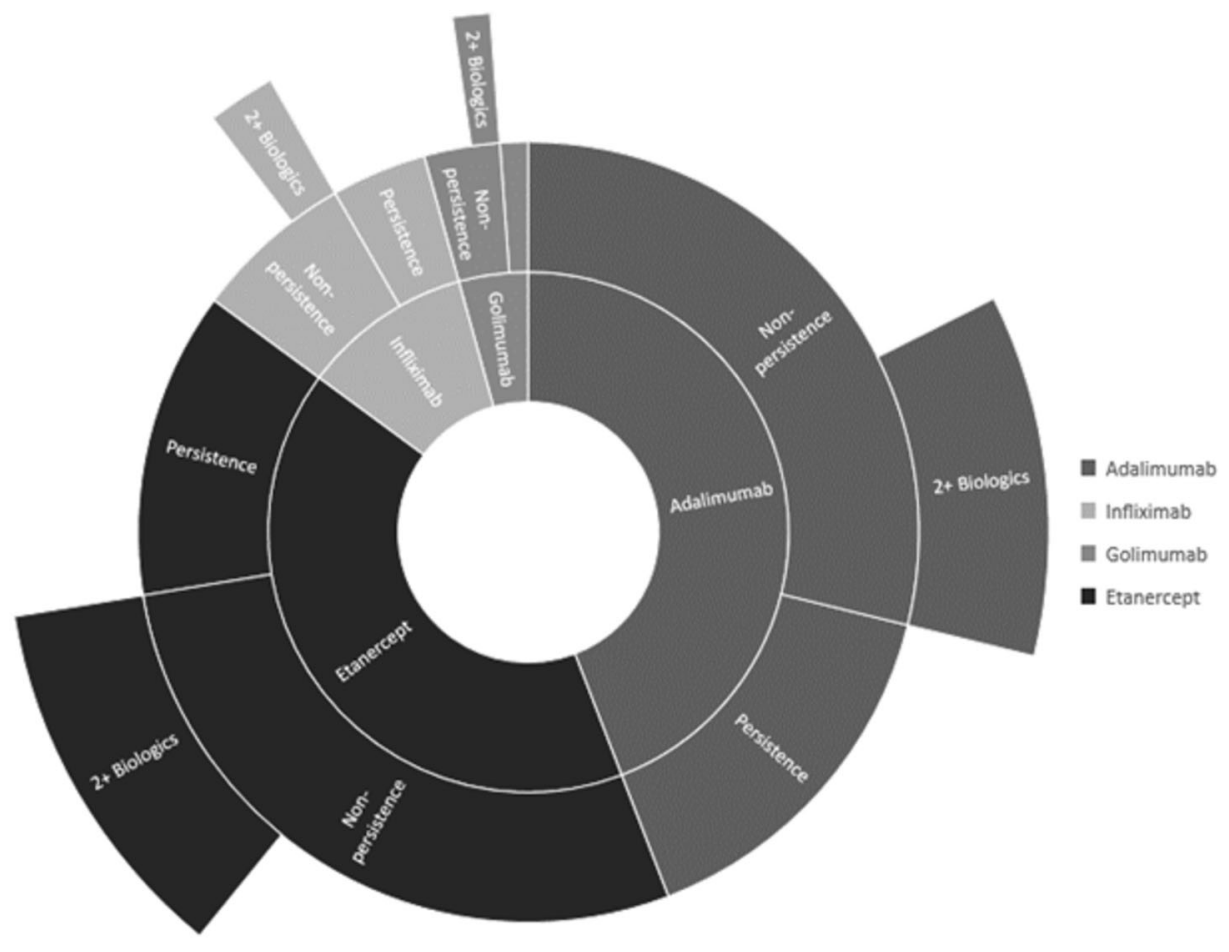

\begin{tabular}{|l|r|r|r|r|}
\hline \multicolumn{2}{|c|}{} & \multicolumn{1}{|c|}{$\begin{array}{c}\text { Persistence } \\
(\mathrm{N}=454)\end{array}$} & \multicolumn{2}{|c|}{$\begin{array}{c}\text { Non-persistence } \\
(\mathrm{N}=918)\end{array}$} \\
\cline { 4 - 5 } & & & $\begin{array}{c}\text { Discontinued (No- } \\
\text { Switch) } \\
(\mathrm{N}=559)\end{array}$ & $\begin{array}{c}\text { Switched to } 2^{\text {nd }} \\
\text { Biologic } \\
(\mathrm{N}=359)\end{array}$ \\
\hline Adalimumab & $605(44.1 \%)$ & $210(34.71 \%)$ & $241(39.83 \%)$ & $154(25.45 \%)$ \\
\hline Certolizumab Pegol & $2(0.1 \%)$ & 0 & 0 & $2(100 \%)$ \\
\hline Infliximab & $145(10.6 \%)$ & $54(37.24 \%)$ & $62(42.76 \%)$ & $29(25.22 \%)$ \\
\hline Golimumab & $59(4.3 \%)$ & $16(27.12 \%)$ & $27(45.76 \%)$ & $16(27.12 \%)$ \\
\hline Etanercept & $561(40.9 \%)$ & $174(31.02 \%)$ & $229(40.82 \%)$ & $158(28.16 \%)$ \\
\hline
\end{tabular}

Fig. 1 Persistence and non-persistence of index TNF inhibitor therapy

\section{Persistence, Discontinuation, and Switching Patterns}

Adalimumab was the index biologic for $44.1 \%$ of patients, followed by etanercept $(40.9 \%)$, infliximab $(10.6 \%)$, golimumab $(4.3 \%)$, and certolizumab pegol (0.1\%) (Fig. 1). During the 2-year follow-up period, $33.1 \%$ of patients $(n=454)$ were persistent on their index TNFi, while $40.7 \%(n=559)$ discontinued their index
TNFi and did not restart a TNFi, and 26.1\% $(n=359)$ switched to a second TNFi. Among the patients who started a second TNFi, 20.1\% $(n=72)$ went on to start a third TNFi (Fig. 2). From 2009 to 2013, the proportion of new TNFi users initiating a second TNFi increased over time from 25.2 to $28.8 \%$ (average for all 4 years $=26.2 \%)$.

Patients prescribed cDMARDs were more likely to be persistent on their index TNFi, while 


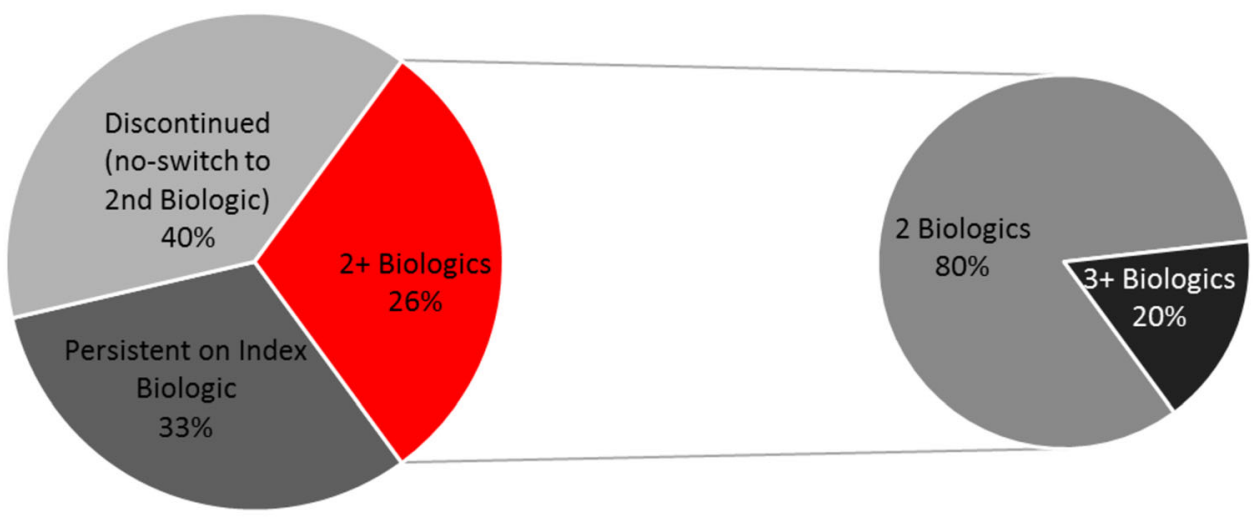

\footnotetext{
- Persistent on Index Biologic

- Discontinued (no-switch to 2nd Biologic)

- $2+$ Biologics

- 3+ Biologics
}

Fig. 2 Persistence, discontinuation, and switching patterns of AS patients initiating TNF inhibitors

Table 1 Baseline patient characteristics

\begin{tabular}{|c|c|c|c|c|}
\hline Variable & Overall $(N=1372)$ & Persistent $(n=454)$ & Discontinued $(N=559)$ & Switched $(n=359)$ \\
\hline \multicolumn{5}{|l|}{ Sex } \\
\hline Male & $846(61.7 \%)$ & $318(70.0 \%)$ & $344(61.5 \%)$ & $184(51.3 \%)$ \\
\hline Female & $526(38.3 \%)$ & $136(30.0 \%)$ & $215(38.5 \%)$ & $175(48.7 \%)$ \\
\hline \multicolumn{5}{|l|}{ Age (years) } \\
\hline Mean & 43.77 & 44.62 & 43.80 & 42.66 \\
\hline $18-24$ & $116(8.5 \%)$ & $27(5.9 \%)$ & $55(9.8 \%)$ & $34(9.5 \%)$ \\
\hline $25-34$ & $231(16.8 \%)$ & $75(16.5 \%)$ & $90(16.1 \%)$ & $66(18.4 \%)$ \\
\hline $35-44$ & $369(26.9 \%)$ & $121(26.7 \%)$ & $151(27.0 \%)$ & $97(27.0 \%)$ \\
\hline $45-54$ & 367 (26.7\%) & $128(28.2 \%)$ & $136(24.3 \%)$ & $103(28.7 \%)$ \\
\hline $55-64$ & $230(16.8 \%)$ & $82(18.1 \%)$ & $96(17.2 \%)$ & $52(14.5 \%)$ \\
\hline $65+$ & $59(4.3 \%)$ & $21(4.6 \%)$ & $31(5.5 \%)$ & $7(1.9 \%)$ \\
\hline \multicolumn{5}{|l|}{ Insurance } \\
\hline Commercial & $1252(91.3 \%)$ & $420(92.5 \%)$ & $492(88.0 \%)$ & $340(94.7 \%)$ \\
\hline Medicare & $69(5.0 \%)$ & $24(5.3 \%)$ & $29(5.2 \%)$ & $7(1.9 \%)$ \\
\hline Medicaid & $51(3.7 \%)$ & $10(2.2 \%)$ & $38(6.8 \%)$ & $12(3.3 \%)$ \\
\hline
\end{tabular}


Table 2 Logistic regression analyses predicting persistent vs. non-persistent to first TNF inhibitor

\begin{tabular}{llllll}
\hline & Regression coefficient & Standard error & Chi-square & Odds ratio (95\% CI) & $\boldsymbol{p}$ value \\
\hline Intercept & -0.9700 & 0.2470 & 15.4200 & & $<0.0001$ \\
Gender (female vs. male) & -0.4563 & 0.1311 & 12.1186 & $0.634(0.49-0.81)$ & 0.0005 \\
Age & 0.00974 & 0.0053 & 3.3297 & $1.010(0.99-1.02)$ & 0.0680 \\
Medicaid & -0.7560 & 0.4170 & 3.2873 & $0.470(0.20-1.06)$ & 0.0698 \\
Medicare & -0.2249 & 0.3030 & 0.5510 & $0.799(0.44-1.44)$ & 0.4579 \\
Use of cDMARD & 0.2633 & 0.1333 & 3.9043 & $1.301(1.00-1.69)$ & 0.0482 \\
Use of opioid & -0.4637 & 0.1226 & 14.3172 & $0.629(0.49-0.80)$ & 0.0002 \\
\hline
\end{tabular}

Table 3 Logistic regression analysis predicting switching TNF inhibitors vs. discontinuing TNF inhibitors

\begin{tabular}{llllll}
\hline & Regression coefficient & Standard error & Chi-square & Odds ratio $(95 \%$ CI) & $\boldsymbol{p}$ value \\
\hline Intercept & -1.2949 & 0.1837 & 49.6609 & & $<0.0001$ \\
Gender (female) & 0.3455 & 0.1390 & 6.1792 & $1.413(1.076-1.855)$ & 0.0129 \\
Medicaid & -0.8016 & 0.3670 & 4.7699 & $0.449(0.218-0.921)$ & 0.0290 \\
Medicare & -1.2866 & 0.4241 & 9.2061 & $0.276(0.120-0.634)$ & 0.0024 \\
Use of cDMARD & 0.3034 & 0.1505 & 4.0631 & $1.354(1.008-1.819)$ & 0.0438 \\
Use of non-opioid analgesics & 0.6615 & 0.1785 & 13.7264 & $1.938(1.366-2.749)$ & 0.0002 \\
\hline
\end{tabular}

females and opioid users were less likely to be persistent on their first TNFi (Table 2). Females and non-opioid analgesic users were more likely to switch to a second TNFi (Table 3).

\section{Gender Comparisons of Persistence, Discontinuation, and Switching Patterns}

Adalimumab was the first TNFi for the majority of patients $(44.6 \%$ males $/ 43.3 \%$ females), followed by etanercept $(40.4 \%$ males $/ 41.6 \%$ females), infliximab $(10.4 \%$ males $/ 10.8 \%$ females), golimumab $(4.6 \%$ males $/ 3.8 \%$ females), and certolizumab pegol (0.0\% males/ $0.4 \%$ females). During the 2-year follow-up period, $32.6 \%$ of males were persistent on their first TNFi, while only $22.8 \%$ of females were persistent. The majority of male $(67.4 \%)$ and female patients $(77.2 \%)$ discontinued their first TNFi during the 2-year follow-up period.
Among those that discontinued their first TNFi, $32.8 \%(n=187)$ of males and $43.6 \%(n=177)$ of females switched to a second TNFi.

\section{DISCUSSION}

To our knowledge, this is the first study to evaluate the persistence, discontinuation, and switching patterns of AS patients in the United States newly initiating TNFi therapy during a 2-year follow-up period. Results from this study highlight the real-world use of a TNFi among patients with AS and found that over two-thirds of AS patients in the US either discontinued their first TNFi or switched to a second TNFi within 2 years from initiating TNFi therapy.

During the 2-year follow-up period, we found that $33.1 \%$ of AS patients were persistent on their index TNFi. Previous studies have evaluated follow-up period of up to 12 months 
and found that between 34 and $48 \%$ of AS patients were persistent with their index TNFi for $\geq 12$ months $[12,13]$. A recent study among US veterans with AS reported that at approximately 3 years, persistence for any TNFi was $<25 \%$ [14]. These findings are consistent with the findings of our study that indicate that TNFi persistence decreases over time.

A total of $66.9 \%$ of AS patients discontinued their index TNFi during the follow-up period in our study. Among the patients who discontinued their first index TNFi, 39.1\% switched to a second TNFi. Walsh and colleagues [13] found that of the $59.4 \%$ of AS patients that discontinued their index TNFi within 12 months, $21.4 \%$ switched to a second TNFi. These results may be attributed to the differences in our study follow-up period or reflect differences in the patient population in the IBM Marketscan database and the Optum Research database.

In our study, we also found that among AS patient receiving a second $\mathrm{TNFi}, 20.1 \%$ had three or more different TNFi prescribed during the 2-year follow-up period. This confirms that many AS patients are cycling through TNFi medications. Since IL-17 inhibitors (IL-17i) were not available during the time period of this study, it is not possible to know if switching to IL-17i would have impacted the treatment patterns observed.

Our study was unique in that we looked at gender differences in TNFi persistence, discontinuation, and switching patterns among AS patients in the United States. During the 2-year follow-up period, approximately one-third $(32.6 \%)$ of males were persistent on their first TNFi, while less than one-fourth $(22.8 \%)$ of females were persistent. These findings are consistent with previous literature indicating that male sex has been associated with a better TNFI drug survival [15-18]. Among males who were non-persistent on their index TNFi, approximately half (53\%) switched to a second TNFi, while the majority (81\%) of females who were non-persistent on their index TNFi switched to a second TNFi.

While this study provides insight into the TNFi treatment patterns of AS patients in the United States, it is important to note that the primary purpose of insurance claims data is administrative and not research. Therefore, there are limitations to using claims data and ICD-9 and ICD-10 codes provided for insurance claims to determine treatment patterns. Medication use can only be analyzed for prescribed medication, which means that over-the-counter medications (such as NSAIDs) would not be included in this analysis.

Additionally, given that the IBM MarketS$\mathrm{can}^{\circledR}$ Research population is composed of patients with commercial, Medicaid, and/or Medicare supplement insurance; there are specific groups (uninsured people, military personnel, and Medicare enrollees without an employer-sponsored supplement plan) that are not represented in our analyses. Approximately one-third of AS patients report disease-related work disability [19]. Given the limitations of the database used in this study, unemployed AS patients would not be included in our analyses since the majority of patients were commercially insured.

In addition, while our study evaluated patterns of persistence and non-persistence, our study could not evaluate the potential reasons for discontinuing or switching treatment. However, previous studies have reported that about $30-36 \%$ of AS patients discontinued or switched their TNFi therapy due to adverse events or lack of treatment effect [12, 17]. Rates of TNFi persistence and non-persistence may also differ for patients that have peripheral manifestations (dactylitis, enthesitis, etc.), which was not accounted for in this study. It is not recommended that patients with purely axial disease be treated with cDMARDs [10], given this the patients in this study receiving cDMARDs may represent a subgroup of patients with peripheral involvement. In addition, several studies have shown that when compared to males, females are less likely to respond to a TNFi $[15,20,21]$. This lack of response may be contributing to the discontinuation rates that we observed among females in our study.

\section{CONCLUSIONS}

This study suggests that approximately twothirds of AS patients newly initiating on a TNFi 
do not remain on the index TNFi 2-years post initiation. In this AS population, the proportion of female patients switching to a second TNFi is higher than the proportion of male patients. More work is needed to understand the reasons for non-persistence and the increasing trend of second-line TNFi use in this population.

\section{ACKNOWLEDGEMENTS}

Funding. Sponsorship for this study and article processing charges were funded by Eli Lilly and Company. All authors had full access to all of the data in this study and take complete responsibility for the integrity of the data and accuracy of the data analysis.

Authorship. All named authors meet the International Committee of Medical Journal Editors (ICMJE) criteria for authorship for this article, had full access to all of the data in this study, take responsibility for the integrity of data and accuracy of the data analysis, and have given their approval for this version to be published.

Disclosures. Theresa Hunter is an employee of Eli Lilly and Company. Krista Schroeder is an employee of Eli Lilly and Company. David Sandoval is an employee of Eli Lilly and Company. Atul Deodhar is an employee of Oregon Health and Science University and has received consulting fees from Eli Lilly and Company.

Compliance with Ethics Guidelines. The study has been performed in accordance with the ethical standards as laid down in the 1964 Declaration of Helsinki and later amendments. This study was a retrospective analysis of administrative claims data and does not contain any human participants or animals. Thus, permission from a research ethics committee was not required and formal informed consent was not obtained. This study was designed and implemented in accordance with the Guidelines for Good Pharmacoepidemiology Practices of the International Society for Pharmacoepidemiology, the Strengthening the Reporting of
Observational Studies in Epidemiology (STROBE) [22] guidelines, and the ethical principles laid down in the 1964 Declaration of Helsinki and its later amendments or comparable ethical standards. This study was a retrospective analysis of administrative claims data and does not contain any human participants or animals. Thus, this study was exempt from an institutional review board.

Data Availability. The datasets generated during and/or analyzed during the current study are available from the corresponding author on reasonable request.

Open Access. This article is distributed under the terms of the Creative Commons Attribution-NonCommercial 4.0 International License (http://creativecommons.org/licenses/ by-nc/4.0/), which permits any noncommercial use, distribution, and reproduction in any medium, provided you give appropriate credit to the original author(s) and the source, provide a link to the Creative Commons license, and indicate if changes were made.

\section{REFERENCES}

1. Sieper J, Braun J, Dougados M, Baeten D. Axial spondyloarthritis. Nat Rev Dis Primers. 2015;1:15013.

2. Boonen A, Brinkhuizen T, Landewe R, et al. Impact of ankylosing spondylitis on sick leave, presenteeism, and unpaid productivity, and estimation of the societal cost. Ann Rheum Dis. 2010;69:1123-8.

3. Kotsis K, Voulgari PV, Dross AA, Carvalho AF, Hyphantis T. Health-related quality of life in patients with ankylosing spondylitis: a comprehensive review. Expert Rev Pharmacoeconomic Outcomes Res. 2014;14:857-72.

4. Braun J, Brandt J, Listing J, et al. Treatment of active ankylosing spondylitis with infliximab: a randomized controlled multicenter trial. Lancet. 2002;359(9313):1187-93.

5. Davis JC Jr, Van Der Heijde D, Braun J, et al. Recombinant human tumor necrosis factor receptor (etanercept) for treating ankylosing spondylitis: a randomized, controlled trial. Arthritis Rheum. 2003;48(11):3230-6. 
6. van der Heijde D, Kivitz A, Schiff MH, et al. Efficacy and safety of adalimumab in patients with ankylosing spondylitis: results of a multicenter, randomized, double-blind, placebo-controlled trial. Arthritis Rheum. 2006;54(7):2136-46.

7. Inman RD, Davis JC Jr, Heijde D, et al. Efficacy and safety of golimumab in patients with ankylosing spondylitis: results of a randomized, double-blind, placebo-controlled, phase III trial. Arthritis Rheum. 2008;58(11):3402-12.

8. Landewe R, Braun J, Deodhar A, et al. Efficacy of certolizumab pegol on signs and symptoms of axial spondyloarthritis including ankylosing spondylitis: 24-week results of a double-blind randomised placebo-controlled phase 3 study. Ann Rheum Dis. 2014;73(1):39-47.

9. Baeten D, Sieper J, Braun J, et al. Secukinumab, an interleukin-17a inhibitor, in ankylosing spondylitis. N Engl J Med. 2015;373(26):2534-48.

10. Ward MM, Deodhar A, Akl EA, et al. American College of Rheumatology/Spondylitis Association of America/Spondyloarthritis Research and Treatment Network 2015 Recommendation for the treatment of ankylosing spondylitis and nonradiographic axial spondyloarthritis. Arthritis Care Res (Hoboken). 2016;68(2):151-66.

11. Lie E, van der Heijde D, Uhlig T, Mikkelsen K, Rodevand E, Koldingsnes W, et al. Effectiveness of switching between TNF inhibitors in ankylosing spondylitis: data from the NOR-DMARD register. Ann Rheum Dis. 2011;70:157-63.

12. Bonafede M, Fox KM, Watson C, Princic N, Gandra SR. Treatment patterns in the first year after initiating tumor necrosis factor blockers in real-world settings. Adv Ther. 2012;29(8):664-74.

13. Walsh J, Adejoro O, Chastek B, Park Y. Treatment patterns of biologics in US patients with ankylosing spondylitis: descriptive analyses from a claims database. J Comp Effect Res. 2018;7(4):369-80.

14. Bekele D, Cheng E, Geier C, Ganuthula K, Walsh J, Dubreuil $\mathrm{M}$, et al. Tumor necrosis factor inhibitor persistence and reasons for discontinuation in US veterans with axial spondyloarthritis [abstract]. Arthritis Rheumatol. 2018; 70 (suppl 10). https:// acrabstracts.org/abstract/tumor-necrosis-factorinhibitor-persistence-and-reasons-for-discontinuation- in-us-veterans-with-axial-spondyloarthritis/. Accessed January 2, 2019.

15. Arends S, Brouwer E, van der Veer E, Groen $\mathrm{H}$, Leijsma MK, Houtman PM, et al. Baseline predictors of response and discontinuation of tumor necrosis factor-alpha blocking therapy in ankylosing spondylitis: a prospective longitudinal observational cohort study. Arthritis Res Ther. 2011;13:94.

16. Kristensen LE, Karlsson JA, Englund M, Petersson IF, Saxne T, Geborek P. Presence of peripheral arthritis and male sex predicting continuation of anti-tumor necrosis factor therapy in ankylosing spondylitis: an observational prospective cohort study from the South Swedish Arthritis Treatment Group Register. Arthritis Care Res. 2010;62:1362-9.

17. Glintborg B, Ostergaard M, Krigh NS, Tarp U, Manilo N, et al. Clinical response, drug survival and predictors thereof in 432 ankylosing spondylitis patients after switching tumor necrosis factor alpha inhibitor therapy: results from the Danish Nationwide DANBIO registry. Ann Rheum. 2013;72:1149-55.

18. Hebeisen M, Neunschwander R, Scherer A, Exer P, Weber U, Tamborrini G, Micheroli R, Wildi L, Zufferey $\mathrm{P}$, et al. Response to tumor necrosis inhibition in male and female patients with ankylosing spondylitis: data from a Swiss Cohort. J Rheumatol. 2018;45(4):506-12.

19. Barlow JH, Wright CC, Williams B, Keta A. Work disability among people with ankylosing spondylitis. Arthritis Care Res. 2001;45(5):424-9.

20. Fagerli KM, Lie E, Heiberg MS, et al. Predictors of ASDAS major improvement in patients with ankylosing spondylitis receiving their first TNF inhibitor. Results from a longitudinal observational study. Arthritis Rheum. 2011;63:204-5.

21. Ramiro S, Machado $P$, Roque $R$, et al. Predictive factors of response at 12 weeks in patients with ankylosing spondylitis starting biological therapies-results from the Portuguese register-Rheuma.Pt. Clin Exp Rheumatol. 2012;30:641-2.

22. von Elm E, Altman DG, Egger $M$, et al. The strengthening the reporting of observational studies in epidemiology (STROBE) statement: guidelines for reporting observational studies. J Clin Epidemiol. 2008;61:344-9. 